\title{
Research on Cooperative Benefit Distribution Mechanism of Pharmaceutical Supply Chain with the Participation of Third-Party Logistics Service Providers
}

\author{
Lijuan Huang, Yingyu Wu*, Lili Liu \\ School of International Pharmaceutical Business, China Pharmaceutical University, Nanjing, China

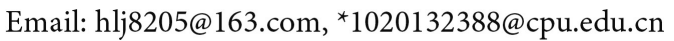

How to cite this paper: Huang, L. J., Wu, Y. Y., \& Liu, L. L. (2022). Research on Cooperative Benefit Distribution Mechanism of Pharmaceutical Supply Chain with the Participation of Third-Party Logistics Service Providers. Advances in Applied Sociology, 12, 34-51.

https://doi.org/10.4236/aasoci.2022.122004

Received: January 24, 2022

Accepted: February 18, 2022

Published: February 21, 2022

Copyright $\odot 2022$ by author(s) and Scientific Research Publishing Inc. This work is licensed under the Creative Commons Attribution International License (CC BY 4.0).

http://creativecommons.org/licenses/by/4.0/

\begin{abstract}
With the cancellation of the policy of "approval of third-party drug logistics business", a large number of powerful enterprises that meet the conditions of modern pharmaceutical logistics have entered the pharmaceutical logistics. In this context, the introduction of third-party logistics service providers into the pharmaceutical supply chain system makes the problem study more practical. A three-level pharmaceutical supply chain operation mode was constructed, which was composed of pharmaceutical manufacturers, pharmaceutical distributors, third-party logistics service providers and retail pharmacies/ hospitals. By using game model, the relevant indicators under independent decision-making and cooperative decision-making can be compared and analyzed. The results show that in a certain range of cooperative cost, the performance index of pharmaceutical supply chain cooperative decision is significantly higher than that of independent decision. On this basis, considering two conditions that every supply chain member can obtain more benefits than that of independent decisions and the relative leadership of supply chain members, the cooperation benefits of pharmaceutical supply chain are reasonably distributed by three-distribution method based on profit distribution factors. Combined with the results, the elements affecting the profit distribution factor are explored, and the value range of the profit distribution factor is continuously reduced until the final result is determined and applied in practice, so as to ensure the stable cooperative operation of the pharmaceutical supply chain system.
\end{abstract}

\section{Keywords}

Third-Party Logistics Service Providers, Pharmaceutical Supply Chain, 
Distribution of Cooperation Benefits, Shapley Value Benefit Distribution Method, Profit Distribution Factor

\section{Introduction}

With the in-depth promotion of national medical reform, two-invoice system, pharmaceutical e-commerce and other related policies, the pharmaceutical logistics market will continue to grow at a rate of no less than $10 \%$ in the next few years (Deng \& Wen, 2019). From the perspective of the operation in pharmaceutical logistics, traditional pharmaceutical wholesale enterprises have monopolized the pharmaceutical logistics field in China for a long time. However, with the cancellation of the policy of "approval of third-party drug logistics business", the signal of deregulatory supervision of pharmaceutical logistics was conveyed in the market, which further released good news for the pharmaceutical third-party logistics market and boosted the market demand for pharmaceutical third-party logistics. Powerful logistics enterprises take advantage of the situation to build pharmaceutical logistics system, and meet the requirements of GSP management, with their own distribution advantages, to share a slice of the pharmaceutical logistics market. S. F. Express, J. D., DHL and other enterprises that meet the requirements of modern pharmaceutical logistics have entered into pharmaceutical logistics and developed into social third-party pharmaceutical logistics. In the context of social encouragement and development of pharmaceutical third-party logistics, it is necessary to introduce third-party logistics service providers into the pharmaceutical supply chain. Therefore, the research object of this paper is "pharmaceutical supply chain with the participation of thirdparty logistics", taking the third-party logistics service provider as one of the stakeholders of pharmaceutical supply chain, studying various performance indicators under independent decision-making and cooperative decision-making of pharmaceutical supply chain members, and studying the reasonable value range of cooperation cost under cooperative decision-making. On this basis, in view of the unreasonable distribution of benefits is often one of the reasons for the failure of cooperation, so, in order to ensure the stable cooperative operation of pharmaceutical supply chain, this paper further studies the mechanism of cooperative benefit distribution among supply chain members, so as to have a certain guiding role in practice.

\section{Literature Review}

With the growing trend of outsourcing, many organizations have outsourced logistics activities to third-party logistics providers to focus on their core competitiveness, thus reducing operating costs, improving service levels and increasing corporate profits. By using empirical analysis, Shi, Zhang, Arthanari, Liu, \& Cheng (2016) verified that the participation of third-party logistics providers in 
the supply chain can bring a mutual benefit situation for themselves and thirdparty logistics users by the means of providing value-added services. Chen, Cai, \& Song (2019) proved that the whole supply chain can benefit from third-party logistics as a supply chain intermediary. Giri \& Sarker (2017) found that thirdparty logistics service has a significant impact on supply chain performance through numerical research. This paper believed that the performance of supply chain composed of monopoly manufacturer, third-party logistics service provider (TPLSP) and multiple independent retailers can be improved through buyback and income sharing contract coordination. Liu, Wu, Lu, \& Pang (2011) applied two-part fee contract to the coordination of the two-level supply chain with the participation of third-party logistics service provider, and finally concluded that pareto improvement of the supply chain system could be realized because the coordination contract had certain flexibility and robustness. Gong, Li, \& Liu (2008) studied the pricing of supply chain system with the participation of thirdparty logistics service providers. Thus, scholars in the domestic and abroad have begun to pay attention to the positive impact of the third-party logistics participation in supply chain and the role that it plays in supply chain management, and have carried out research on the coordination and pricing of supply chain system under the participation of third-party logistics.

In the field of pharmaceutical supply chain, Volland, Fügener, \& Schoenfelder (2017) discussed the importance of logistics distribution capability in pharmaceutical supply chain, saying that it could improve the profit of supply chain members without affecting the quality of patients' medication. Chen, Zhang, \& Gu (2020) proved that improving logistics distribution capacity within a certain range can improve the profits of pharmaceutical distribution enterprises, as well as the order quantity and the profits of supply chain members through algorithm analysis. However, these two literatures do not emphasize the fact that logistics distribution is provided by the third-party logistics service providers.

To the distribution of supply chain cooperation benefits, Sha \& Zheng (2021) insisted that it not only determines the coordination of supply chain, but also determines the development of supply chain. At present, there is little research on the distribution of cooperative benefits among members of pharmaceutical supply chain by domestic and foreign scholars. However, many scholars have paid attention to and studied other industries. The differences lie in different methods of profit distribution and different influencing factors when considering the distribution. Among them, Shapley proposed the Shapley value method model in 1953, which was widely adopted by scholars due to its simplicity of calculation and uniqueness (Hafezalkotob, Khodabakhsh, Saghaei, \& Eshghipour, 2018; Zhou, Cui, Huang, Kang, \& Zhang, 2021; Gao, Yang, \& Liu, 2017; Liang \& Li, 2013). Huang, Mi, \& Kang (2021) further verified the superiority and effectiveness of this method through two application examples of target recognition and fault diagnosis. However, the traditional Shapley value takes marginal contribution as the only factor affecting the distribution of benefits, ignoring the 
impact of investment, risk and other factors. Therefore, many scholars have improved it in practical application and applied the revised Shapley value to realize the reasonable distribution of benefits in supply chain partners (Zheng, Zhang, Chen, \& Liu, 2011; Yang, Sun, Liu, \& Hao, 2017; Xu, Peng, Yang, \& Chen, 2018; Yang, Liu, \& Li, 2017; Zhou, 2017). In the field of pharmaceutical supply chain, Di, Zhao, \& Yu (2017) optimized the supply chain management of pharmaceutical distribution enterprises by adopting the modified Shapley value benefit distribution method on the basis of considering the input and risk factors. Lyu \& Liu (2010) also used the profit distribution method based on profit distribution factors to distribute the cooperation benefits of pharmaceutical supply chain.

To sum up, at present, few domestic and abroad scholars have introduced the third-party logistics into the pharmaceutical supply chain for research, which is obviously inconsistent with the current situation of pharmaceutical circulation. The pharmaceutical supply chain members studied in this paper include thirdparty logistics service providers. As for the distribution of cooperative benefits in pharmaceutical supply chain, no matter how Shapley valued distribution method, modified Shapley value distribution method or distribution method based on profit distribution factor is adopted in the existing literature, the dominant position of stakeholders in the supply chain is not taken into account, nor the occurrence of cooperation costs in cooperative decision-making. Therefore, In this paper, on the one hand, in view of information asymmetry and egoism, the occurrence of cooperation cost in cooperative decision-making is considered. On the other hand, considering the position of individual's interest subject in the supply chain, the third distribution method based on profit distribution factor is used to study the problem of supply chain cooperation profit distribution.

\section{Modeling and Analysis}

Since the implementation of the "Two-invoice system" policy, the original pharmaceutical ecosystem has been reconstructed, greatly simplifying the intermediate circulation links of drugs and medical devices, and making the whole circulation process relatively flat (Deng \& Wen, 2018; Zheng, Song, Wu, Zhang, \& Gao, 2018). Under this circumstance, the pharmaceutical supply chain model in this study is shown as Figure 1. In Figure 1, the supply chain members operate as follows. Above all, pharmaceutical manufacturers sell their drugs or medical devices to pharmaceutical distributors at the ex-factory price of the enterprise, Pharmaceutical distributors then sell them to retail pharmacies and hospitals at the wholesale price, and retail pharmacies and hospitals finally sell to patients at the retail price. Third-party logistics service providers are in charge of the whole circulation and distribution of drugs or medical device. The solid line in Figure 1 represents medical entity flow and the dotted line represents information flow.

In this mode of operation, it is assumed that a three-level pharmaceutical supply chain system consists of a pharmaceutical manufacturer, a pharmaceutical distributor, a third-party logistics service provider and a retail pharmacy or 


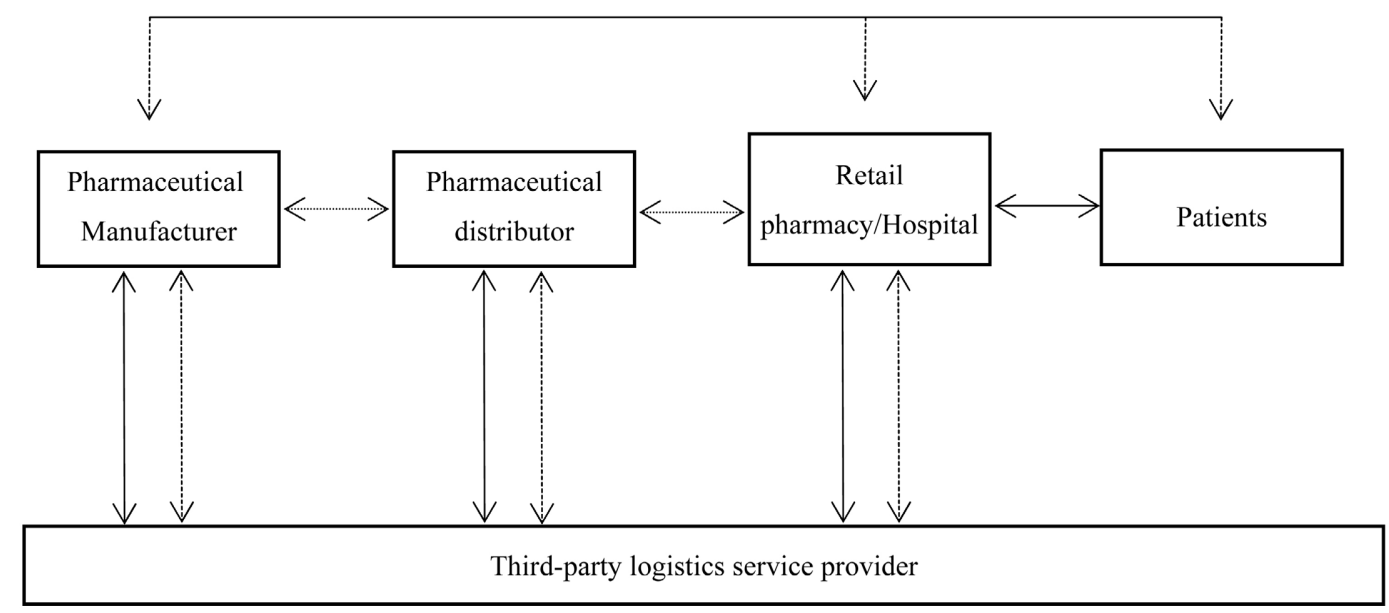

Figure 1. Operation mode of pharmaceutical supply chain.

hospital. Every stakeholder in this chain pursues the their own maximum interests as the ultimate goal. For this reason, parameters are set as follows.

$C_{m}$ : Unit production cost incurred by pharmaceutical manufacturers

$P_{m}$ : Unit ex-factory price made by the pharmaceutical manufacturer on the basis of the production cost and national policies

$C_{d}$ : Unit management cost incurred by pharmaceutical distributors

$P_{d}$ : Unit wholesale price by pharmaceutical distributors

$C_{l}$ : Unit distribution and service costs incurred by third-party logistics service providers

$P_{l}$ : Unit distribution and service price determined by third-party logistics service providers

$C_{r}$ : Unit selling cost incurred by retail pharmacies or hospitals

$P_{r}$ : Unit selling price incurred by retail pharmacies or hospitals

$Q$. Patients' market demand for drugs and medical devices

$\pi_{m}$ : Expected profit of pharmaceutical manufacturers

$\pi_{d}$ : Expected profit of pharmaceutical distributors

$\pi_{l}$ : Expected profit of third-party logistics service providers

$\pi_{r}$ : Expected profit of retail pharmacies or hospitals

$\pi_{t}$ : Overall expected profit of pharmaceutical supply chain system.

\subsection{Modeling}

The Pharmaceutical market demand involves many factors, such as market price, medical insurance policy, service level, consumer payment ability, consumer's utility, brand loyalty and so on (Hou \& Yang, 2019; Guan \& Huang, 2021; Yang \& Hou, 2019; Lai \& Nie, 2021; Li, Dan, Zhou, \& Wang, 2019). In this paper, we assume that the market demand is sensitive to the sales price of pharmaceutical market and it is affected by the logistics distribution and service level provided by the third party logistics. So the following linear demand function is constructed in reference (Xu, Yu, \& Zhang, 2006).

$$
Q\left(P_{r}, S\right)=Q_{0}-a P_{r}+\lambda S
$$


In this function, $P_{r}$ is the pharmaceutical retail price sold by retail pharmacies or hospitals. $S$ is logistics distribution and service level provided by third-party logistics service providers. They are regarded as endogenous variables affecting market demand. $Q_{0}, a$ and $\lambda$ are constants greater than $0 . Q_{0}$ indicates the basic market demand of patients. a refers to the sensitivity of market demand to retail price, that is, the increase or decrease of market demand when the unit retail price descends or rises under certain cases of logistics distribution and service level. $\lambda$ refers to the sensitivity of market demand to the logistics distribution and service level provided by third-party logistics service provider. That is to say, the increase or decrease of market demand when the logistics distribution and service level provided by third-party logistics service provider increases or decreases by one unit in the case that the retail price remains unchanged.

For third-party logistics service providers, with the improvement of logistics distribution and service level, the logistics cost naturally increases. In view of this, according to the research of Xu, Yu, \& Zhang (2006), the unit logistics cost was expressed as a quadratic expense function $C_{l}(s)=\frac{1}{2} \eta S^{2} . \eta$ is a constant greater than 0 , indicating the elastic coefficient of logistics cost changing with logistics distribution and service level.

In order to closely follow the research objectives of this paper and make the model easy to analyze, the main assumptions of this paper are as follows:

Assumption 1: the order quantity of retail terminal is equal to the market demand of drugs, regardless of drug inventory, drug out of stock.

Assumption 2: the same third-party logistics service provider is responsible for transportation and distribution and provides logistics services from the pharmaceutical manufacturer to the pharmaceutical distributor to the retail pharmacy or the hospital, and the expenses are paid by the pharmaceutical distributor.

Assumption 3: in the supply chain, only the pricing and demand of a single drug are considered, and the market conditions of other drugs that can replace this variety are not considered.

Assumption 4: the market is in a state of complete competition, and the relevant parameters such as ex-factory price, wholesale price, logistics distribution price, retail price and relevant pharmaceutical cost meet $P_{r}>P_{d}>P_{m}, P_{m}>C_{m}$, $P_{d}>P_{m}+P_{l}+C_{d}, P_{l}>C_{l}, P_{r}>P_{d}+C_{l}$.

Based on the above assumptions, the profit function of each enterprise in the pharmaceutical supply chain studied in this paper when making independent decisions is expressed as follows:

1) Profit of the pharmaceutical manufacturer: $\pi_{m}=\left(P_{m}-C_{m}\right) \times Q$

2) Profit of the pharmaceutical distributor: $\pi_{d}=\left(P_{d}-C_{d}-P_{m}-P_{l}\right) \times Q$

3) Profit of the third party logistics service provider: $\pi_{l}=\left(P_{l}-C_{l}\right) \times Q$

4) Profit of the retail pharmacy or hospital: $\pi_{r}=\left(P_{r}-C_{r}-P_{d}\right) \times Q$

The overall profit of the supply chain is expressed as follows when cooperation decisions are made and cooperation costs are not considered:

$\pi_{t}=\left(P_{r}-C_{r}-C_{d}-C_{l}-C_{m}\right) \times Q$. 


\subsection{Modeling Solution and Analysis}

In this part, through the game model constructed in 3.1, the performance indicators of pharmaceutical supply chain members under independent decisionmaking and cooperative decision-making are calculated respectively, and the indicators under the two decision-making are compared and analyzed. On this basis, the cooperative cost under cooperative decision is set, and according to the condition that the net benefit brought by cooperative decision is greater than 0 compared with independent decision, the range of cooperative cost is obtained. In other words, as long as the cooperation cost is within this value range, the total benefit of the supply chain under cooperative decision will always exceed that under independent decision.

\subsubsection{Independent Decision Model}

Under the case of independent decision-making, the members of the pharmaceutical supply chain have inconsistent goals, and strive to maximize their own interests, regardless of cooperation and coordination of the overall supply chain. According to the Stackelberg's game idea, the reverse derivation method is taken to solve the interest equilibrium model of pharmaceutical supply chain, and then the following results are obtained.

The optimized sales quantity in the pharmaceutical supply chain under independent decision-making is:

$$
Q^{*}=\frac{Q_{0}-a\left(C_{r}+C_{d}+C_{m}\right)}{16}+\frac{\lambda^{2}}{32 a \eta}
$$

The optimized ex-factory price of the pharmaceutical manufacturer is:

$$
P_{m}^{*}=\frac{Q_{0}-a\left(C_{r}+C_{d}-C_{m}\right)}{2 a}+\frac{\lambda^{2}}{4 a^{2} \eta}
$$

The optimized logistics distribution and service price of the third-party logistics service provider is:

$$
P_{l}^{*}=\frac{Q_{0}-a\left(C_{r}+C_{d}+C_{m}\right)}{4 a}+\frac{5 \lambda^{2}}{8 a^{2} \eta}
$$

The optimized logistics distribution and service level provided by the thirdparty logistics service provider is:

$$
S^{*}=\frac{\lambda}{a \eta}
$$

The optimized wholesale price of the pharmaceutical distributor is:

$$
P_{d}^{*}=\frac{7 Q_{0}+a\left(C_{d}+C_{m}\right)-7 a C_{r}}{8 a}+\frac{15 \lambda^{2}}{16 a^{2} \eta}
$$

The optimized retail price of the retail pharmacy or hospital is:

$$
P_{r}^{*}=\frac{15 Q_{0}+a\left(C_{m}+C_{d}+C_{r}\right)}{16 a}+\frac{31 \lambda^{2}}{32 a^{2} \eta}
$$


Thus, the optimized profit of the pharmaceutical manufacturer when making independent decisions is:

$$
\pi_{m}^{*}=\frac{\left[Q_{0}-a\left(C_{r}+C_{d}+C_{m}\right)+\frac{\lambda^{2}}{2 a \eta}\right]^{2}}{32 a}
$$

The optimized profit of the third-party logistics service provider when making independent decisions is:

$$
\pi_{l}^{*}=\frac{\left[Q_{0}-a\left(C_{r}+C_{d}+C_{m}\right)+\frac{\lambda^{2}}{2 a \eta}\right]^{2}}{64 a}
$$

The optimized profit of the pharmaceutical distributor when making independent decisions is:

$$
\pi_{d}^{*}=\frac{\left[Q_{0}-a\left(C_{r}+C_{d}+C_{m}\right)+\frac{\lambda^{2}}{2 a \eta}\right]^{2}}{128 a}
$$

The optimized profit of the retail terminal when making independent decision is:

$$
\pi_{r}^{*}=\frac{\left[Q_{0}-a\left(C_{r}+C_{d}+C_{m}\right)+\frac{\lambda^{2}}{2 a \eta}\right]^{2}}{256 a}
$$

Conclusion is that the overall profit contributed by every member of the pharmaceutical supply chain when making independent decisions with the participation of third party logistics service provider is:

$$
\pi_{t}^{*}=\pi_{m}^{*}+\pi_{l}^{*}+\pi_{d}^{*}+\pi_{r}^{*}=\frac{15\left[Q_{0}-a\left(C_{r}+C_{d}+C_{m}\right)+\frac{\lambda^{2}}{2 a \eta}\right]^{2}}{256 a}
$$

\subsubsection{Cooperative Decision Model}

Under cooperative decision model, pharmaceutical manufacturers, distributors, retail pharmacies or hospitals and third-party logistics service providers share the same supply chain system in order to maximize the overall benefits of the supply chain. In order to make the assure of the cooperation among certain cooperation members of the supply chain, cooperative cost must occur among members, such as communication cost, information cost, time cost, operation cost, etc. It is assumed that the unit cooperation cost is $C_{0}$ when cooperation decisions are made, without discussing who should share the cost. Thus, overall profit function of pharmaceutical supply chain system is written as: $\widehat{\pi_{t}}=\left(P_{r}-C_{m}-C_{d}-C_{l}-C_{r}-C_{0}\right) \times Q$.

Substitute $P_{r}=\frac{Q_{0}+\lambda S-Q}{a}$ into the above formula to achieve the overall profit function of the pharmaceutical supply chain is: 


$$
\widehat{\pi_{t}}=\left(\frac{Q_{0}+\lambda S-Q}{a}-C_{m}-C_{d}-C_{l}-C_{r}-C_{0}\right) \times Q
$$

The second-order partial derivative of the sales quantity $Q$ in Equation (13) is obtained: $\frac{\partial^{2} \widehat{\pi_{t}}}{\partial Q^{2}}<0$. So, when $\frac{\partial \widehat{\pi_{t}}}{\partial Q}=0$, it can be solved:

$$
\widehat{Q^{*}}=\frac{Q_{0}+\lambda S-a\left(C_{m}+C_{d}+C_{r}+C_{0}+C_{l}\right)}{2}
$$

At this time, the overall profit of the pharmaceutical supply chain system is maximized.

At the same time, Substitute $C_{l}(s)=\frac{1}{2} \eta S^{2}$ into Formula (13) and get:

$$
\widehat{\pi_{t}}=\left(\frac{Q_{0}+\lambda S-Q}{a}-C_{m}-C_{d}-\frac{1}{2} \eta S^{2}-C_{r}-C_{0}\right) \times Q .
$$

The second-order partial derivative of $S$ in Formula (15) is obtained: $\frac{\partial^{2} \widehat{\pi_{t}}}{\partial S^{2}}<0$. So, when $\frac{\partial \widehat{\pi_{t}}}{\partial S}=0$, there exists the optimized logistics distribution and service level, and the solution is as follows: $\widehat{S^{*}}=\frac{\lambda}{a \eta}$.

Substitute $\widehat{S^{*}}=\frac{\lambda}{a \eta}$ into Formula (14), it is solved the optimized sales quantity under cooperative decision is:

$$
\widehat{Q^{*}}=\frac{Q_{0}-a\left(C_{m}+C_{d}+C_{r}+C_{0}\right)}{2}+\frac{\lambda^{2}}{4 a \eta}
$$

At this point, the retail price of the retail pharmacy or hospital is:

$$
\widehat{P_{r}^{*}}=\frac{Q_{0}+a\left(C_{m}+C_{d}+C_{r}+C_{0}\right)}{2 a}+\frac{3 \lambda^{2}}{4 a^{2} \eta}
$$

The optimized profit of the overall supply chain is:

$$
\widehat{\pi_{t}^{*}}=\frac{\left[Q_{0}-a\left(C_{r}+C_{d}+C_{m}\right)+\frac{\lambda^{2}}{2 a \eta}-a C_{0}\right]^{2}}{4 a}
$$

\subsubsection{Comparison Analysis between Independent Decision Making and Cooperative Decision Making}

Through the method of model solution under independent decision-making and cooperative decision-making, some indicators are obtained as shown in Table 1.

It is easy to find that the optimized logistics distribution and service level provided by the third-party logistics under the two decisions-making are equal, and the more sensitive the market demand is to this index (that is to say $\lambda$ is greater), the higher the logistics distribution and service level provided by the thirdparty logistics. At this time, whether the pharmaceutical sales quantity, retail 
Table 1. Comparison of some indicators under cooperative decision and independent decision.

\begin{tabular}{ccc}
\hline & Independent decision & Cooperative decision \\
\hline$S$ & $\frac{\lambda}{a \eta}$ & $\frac{\lambda}{a \eta}$ \\
$Q$ & $\frac{Q_{0}-a\left(C_{r}+C_{d}+C_{m}\right)}{16}+\frac{\lambda^{2}}{32 a \eta}$ & $\frac{Q_{0}-a\left(C_{m}+C_{d}+C_{r}+C_{0}\right)}{2}+\frac{\lambda^{2}}{4 a \eta}$ \\
$P_{r}$ & $\frac{15 Q_{0}+a\left(C_{m}+C_{d}+C_{r}\right)}{16 a}+\frac{31 \lambda^{2}}{32 a^{2} \eta}$ & $\frac{Q_{0}+a\left(C_{m}+C_{d}+C_{r}+C_{0}\right)}{2 a}+\frac{3 \lambda^{2}}{4 a^{2} \eta}$ \\
$\pi_{t}$ & $\frac{15\left[Q_{0}-a\left(C_{r}+C_{d}+C_{m}\right)+\frac{\lambda^{2}}{2 a \eta}\right]^{2}}{256 a}$ & $\frac{\left[Q_{0}-a\left(C_{r}+C_{d}+C_{m}\right)+\frac{\lambda^{2}}{2 a \eta}-a C_{0}\right]^{2}}{4 a}$
\end{tabular}

price or the total profit of the pharmaceutical supply chain will all increase. The sensitivity of market demand to the third-party logistics distribution has positive relation with logistics distribution service level, sales quantity, retail price and the total profit of supply chain.

It can also be seen from the table that when the cooperation cost $C_{0}=0$, the sales quantity under the cooperation decision is 8 times of that under the independent decision. The price under the cooperation decision is less than that under the independent decision. But the overall profit of the supply chain under the cooperation decision is 4.267 times of that under the independent decision. Obviously, the performance indicators under cooperative decision-making are significantly higher than those under independent decision-making. Mutual benefit is the future main-stream road of pharmaceutical supply chain. However, in fact, due to information asymmetry and the egoism (that is, to meet the maximization of their own interests rather than the overall interests), it is difficult to achieve the situation that members of the supply chain can consciously cooperate without any cost. In other words, for members of the pharmaceutical supply chain, if they want to achieve cooperation with each other, one condition is that the cooperation cost will occur. Second, after cooperation, the benefits of each supply chain member are greater than those earned when independent decisions are made. With the rising cooperation cost, the retail price continues to rise under the cooperation decision and the sales quantity continues to decline, but the overall profit of the supply chain is getting lower and lower (see Table 1 available under the cooperation decision). With the increase of $C_{0}$ leading to total profit of the supply chain under cooperative decision-making lower than that under independent decision-making, cooperation is meaningless and inefficient, thus cooperation will not occur at this time.

So, when the supply chain members make cooperative decisions, $C_{0}$ has a certain value range, which should be the interval value that the net income brought by cooperative decision-making should be greater than 0 compared with inde- 
pendent decision-making, that is, the value when $\hat{\pi}_{t}^{*}-\pi_{t}^{*}>0$ is satisfied.

According to the total profit formula of supply chain under independent decision and cooperative decision in Table 1 , we set $B=Q_{0}-a\left(C_{r}+C_{d}+C_{m}\right)+\frac{\lambda^{2}}{2 a \eta}$, $B$ is a constant greater than 0 .

Then the total profit of supply chain under independent decision-making can be simplified as $\pi_{t}^{*}=\frac{15 B^{2}}{256 a}$.

The total profit of supply chain under cooperative decision-making can be simplified as $\hat{\pi}_{t}^{*}=\frac{\left(B-a C_{0}\right)^{2}}{4 a}$.

Let $\Delta \pi_{t}=\hat{\pi}_{t}^{*}-\pi_{t}^{*}=\frac{\left(B-a C_{0}\right)^{2}}{4 a}-\frac{15 B^{2}}{256 a}=\frac{49 B^{2}-128 B a C_{0}+64 a^{2} C_{0}^{2}}{256 a}, C_{0}$ as a variable, sort it out as: $\Delta \pi_{t}=\frac{1}{4} a C_{0}^{2}-\frac{1}{2} B C_{0}+\frac{49}{256 a} B^{2}$.

The parabola of net income changing with $C_{0}$ is shown in Figure 2.

When $\Delta \pi_{t}=0, C_{0}^{1}=\frac{(8-\sqrt{15}) B}{8 a}, C_{0}^{2}=\frac{(8+\sqrt{15}) B}{8 a}$.

As shown in Figure 2, combined with the solutions obtained, it can be known that:

1) When $C_{0} \in\left(0, \frac{(8-\sqrt{15}) B}{8 a}\right), \Delta \pi_{t}>0$ (the shaded area in Figure 2), this interval value is the value range satisfying the overall benefit under cooperative decision is greater than that under independent decision.

2) When $C_{0} \in\left[\frac{(8-\sqrt{15}) B}{8 a}, \frac{(8+\sqrt{15}) B}{8 a}\right], \Delta \pi_{t} \leq 0 \quad\left(C_{01}, C_{02}\right.$, parabola and the horizontal axis of the part in Figure 2), members of the pharmaceutical supply chain do not choose to make cooperative decisions.

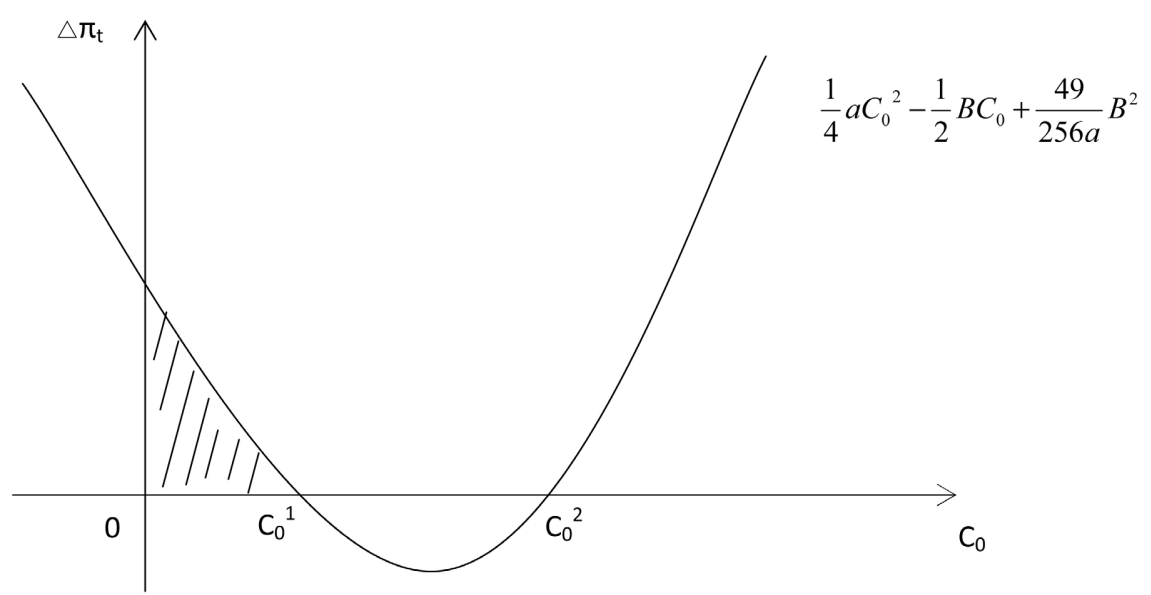

Figure 2. Parabolic curve of net income as $\mathrm{C}_{0}$ change. 
3) When $C_{0} \in\left(\frac{(8+\sqrt{15}) B}{8 a}, \infty\right)$, not in line with the actual situation, undesirable.

From the above analysis, it can be vividly seen that when the unit cooperation cost $C_{0}$ meets case (1), supply-chain members are incentive to choose cooperative decision as the result of the total income of the supply chain in this case exceeding the total income of independent decision. In order to make permanent cooperation among supply chain members, it is also essential to make every supply chain member achieve greater benefit than that of independent decision. So, it is necessary to reasonably allocate the total income of the supply chain. The fourth part of this paper will focus on the distribution of total profit of pharmaceutical supply chain under cooperative decision-making. Through a certain benefit distribution mechanism, the total profit is distributed to the pharmaceutical manufacturer, the pharmaceutical distributor, the third-party logistics service provider and the pharmaceutical retailer, so that each pharmaceutical supply chain member can obtain higher benefit than that under independent decision-making, so as to ensure the stable cooperation operation of pharmaceutical supply chain.

\section{Allocation of Total Income in Pharmaceutical Supply Chain under Cooperative Decision-Making}

This part mainly discusses the distribution of the total income of the pharmaceutical supply chain under the cooperative decision-making at that time on the basis of $C_{0} \in\left(0, C_{0}^{1}\right)$. It mainly adopts the cubic distribution method based on the profit distribution factor to distribute. For the pharmaceutical supply chain in this paper, pharmaceutical manufacturers are in the leading position, followed by distributors and third-party logistics service providers, and retail pharmacies or hospitals are the last. Firstly, the total income is distributed between drug manufacturers and non-drug manufacturers for the first time. The second distribution is conducted among non-drug manufacturers, taking drug distributors and third-party logistics service providers as a whole. The second distribution is conducted between the whole and retail pharmacies or hospitals. Eventually, the last distribution is made between pharmaceutical distributors and third-party logistics service providers. The core principle of distribution is to always ensure that the allocated cooperative income exceeds the income under the independent decision-making of supply chain members while considering the position of members in the supply chain.

In the first distribution, considering the relative dominant position of pharmaceutical manufacturers, the income factor of distribution is higher than that of non-drug manufacturers. In the second round, considering the relative dominant position of pharmaceutical distributors and third-party logistics service providers, the income factor of distribution is higher than that of retail pharmacies or hospitals. In the third round, as the relative dominant position of pharmaceutical dis- 
tributors, the income factor of distribution is higher than that of third-party logistics service providers. So far, three allocations have been completed. This allocation method not only makes the assure of the reasonable income of supply chain members, but also considers the relative leadership of supply chain members in order to contribute to the permanent stable operation of supply chain system, which is conducive to the cooperation among supply chain members.

\subsection{The First Allocation of the Overall Income}

We assume that the distribution factor of the overall income of the supply chain is $e_{x}$ (where $x=1,2$ and 3 represent the distribution factors of the first, second and third distribution respectively, and $0<e_{x}<1$ ). In the first round, the profit distribution factor allocated to pharmaceutical manufacturers is $e_{1}$, thus the profit which is allocated to non-pharmaceutical manufacturers is $1-e_{1}$. In view of the leading position of the pharmaceutical manufacturer, $e_{1}>1-e_{1}$, so $e_{1}>$ $1 / 2$. Other conditions are as follows:

$$
\left\{\begin{array}{l}
e_{1} \times \hat{\pi}_{t}^{*}>\pi_{m}^{*} \\
\left(1-e_{1}\right) \times \hat{\pi}_{t}^{*}>\pi_{l}^{*}+\pi_{d}^{*}+\pi_{r}^{*} \\
\frac{1}{2}<e_{1}<1
\end{array}\right.
$$

As for (19), taking $e_{1}$ as a variable, the value of $e_{1}$ is:

$$
\left(\frac{B^{2}}{8\left(B-a C_{0}\right)^{2}}, 1-\frac{7 B^{2}}{64\left(B-a C_{0}\right)^{2}}\right) \cap\left(\frac{1}{2}, 1\right)
$$

The pharmaceutical manufacturer obtained the profit $e_{1} \times \hat{\pi}_{t}^{*}$ through the first distribution. The value $e_{1}$ is shown in the Formula (20). The pharmaceutical manufacturer will use its leading position in the supply chain to make $e_{1}$ move to the right as much as possible to obtain more income. However, in order to stabilize the whole supply chain, attention should be paid to certain degree.

\subsection{The Second Allocation of the Overall Income}

The income of $e_{1}$ share is distributed to the pharmaceutical manufacturer by means of the first distribution. As for pharmaceutical non-manufacturers, the remaining undistributed total income is $\left(1-e_{1}\right) \times \hat{\pi}_{t}^{*}$. The second distribution occurs among the pharmaceutical distributor, the third-party logistics service provider and the retail pharmacy or hospital. The distributor and the third-party logistics service provider are regarded as a whole. It is assumed that the profit distribution factor allocated to the whole is $e_{2}$, thus the profit allocated to the retail pharmacy or hospital is $1-e_{2}$. In the light of the relative dominance of the whole, it is required to satisfy $e_{2}>1-e_{2}$, so $e_{2}>1 / 2$. Other conditions are as follows:

$$
\left\{\begin{array}{l}
e_{2} \times\left(1-e_{1}\right) \times \hat{\pi}_{t}^{*}>\pi_{l}^{*}+\pi_{d}^{*} \\
\left(1-e_{2}\right) \times\left(1-e_{1}\right) \times \hat{\pi}_{t}^{*}>\pi_{r}^{*} \\
\frac{1}{2}<e_{2}<1
\end{array}\right.
$$


As for Formula (21), at this time, the first allocation has been completed. $e_{1}$ is a known number, and taking $e_{2}$ as a variable, the value of $e_{2}$ is expressed as:

$$
\left(\frac{3 B^{2}}{32\left(B-a C_{0}\right)^{2}\left(1-e_{1}\right)}, 1-\frac{B^{2}}{64\left(B-a C_{0}\right)^{2}\left(1-e_{1}\right)}\right) \cap\left(\frac{1}{2}, 1\right)
$$

It can be seen that by means of the second distribution, the pharmaceutical distributor and the third-party logistics service provider obtain the profits $e_{2} \times\left(1-e_{1}\right) \times \hat{\pi}_{t}^{*}$, the retail pharmacy or hospital obtain the profits $\left(1-e_{2}\right) \times\left(1-e_{1}\right) \times \hat{\pi}_{t}^{*}$. The value $e_{2}$ is shown in Equation (22) above. Similarly, the pharmaceutical distributor and the third-party logistics service provider will use their leading position in the supply chain to make $e_{2}$ move to the right as much as possible to obtain more income.

\subsection{The Third Allocation of the Overall Income}

The last distribution occurs between the pharmaceutical distributor and the third-party logistics service provider to allocate the remaining profit of $e_{2} \times\left(1-e_{1}\right) \times \hat{\pi}_{t}^{*}$. We assume that the profit distribution factor allocated to the pharmaceutical distributor is $e_{3}$, so the profit allocated to the third-party logistics service provider is $1-e_{3}$. In view of the relative dominant position of the distributor, $e_{3}>1-e_{3}$, so $e_{3}>1 / 2$. Other conditions are listed as follows:

$$
\left\{\begin{array}{l}
e_{3} \times e_{2} \times\left(1-e_{1}\right) \times \hat{\pi}_{t}^{*}>\pi_{d}^{*} \\
\left(1-e_{3}\right) \times e_{2} \times\left(1-e_{1}\right) \times \hat{\pi}_{t}^{*}>\pi_{l}^{*} \\
\frac{1}{2}<e_{3}<1
\end{array}\right.
$$

For Formula (23), at this time, the second allocation has finished, $e_{1}$ and $e_{2}$ are known parameters, and taking $e_{3}$ as a variable, the value of $e_{3}$ is solved as follows:

$$
\left(\frac{B^{2}}{32\left(B-a C_{0}\right)^{2}\left(1-e_{1}\right) e_{2}}, 1-\frac{B^{2}}{16\left(B-a C_{0}\right)^{2}\left(1-e_{1}\right) e_{2}}\right) \cap\left(\frac{1}{2}, 1\right)
$$

Through three distributions of the overall profit, the pharmaceutical manufacturer earned the profit $e_{1} \times \hat{\pi}_{t}^{*}$, the pharmaceutical distributor earned the profit $e_{3} \times e_{2} \times\left(1-e_{1}\right) \times \hat{\pi}_{t}^{*}$, the third-party logistics service provider earned the profit $\left(1-e_{3}\right) \times e_{2} \times\left(1-e_{1}\right) \times \hat{\pi}_{t}^{*}$, the retail pharmacy or hospital earned the profit $\left(1-e_{2}\right) \times\left(1-e_{1}\right) \times \hat{\pi}_{t}^{*}$. The profits earned by all types of supply chain members exceed the profits under independent decision-making, making the assure of the basic premise of cooperative operation of the supply chain. The calculated $e_{1}, e_{2}$ and $e_{3}$ allocation factors have a value range, and their specific values will be affected by some elements, such as the bargaining power of supply chain members. For the pharmaceutical supply chain, if it is a scarce drug, pharmaceutical manufacturers monopolize the market or the manufacturers are extremely limited, then the pharmaceutical manufacturers are in an absolutely dominant position. As a result, they have strong bargaining ability, and obtain the distribu- 
tion factor will naturally be higher. If it is a common drug produced by many manufacturers at the same time and curative effect is the same, the distributors and retailers will have stronger bargaining power, so the distribution of interests tend to incline to distributors and retailers.

\section{Research Findings with Analysis}

This paper studies the cooperative benefit distribution mechanism of pharmaceutical three-level supply chain with the participation of third-party logistics service providers. By means of constructing the game model and solving the model, research findings indicate that the optimized logistics distribution and service level offered by the two decisions is the same. To the third-party logistics service provider, the sensitivity of market demand to logistics distribution and service level is positive related to the amount of sales, retail price and the overall profit of the supply chain. So, when the market demand is more sensitive to logistics distribution and service level, it is more necessary to provide higher logistics distribution and service level, in order to make the whole supply chain can obtain higher sales and more profits. Besides, without considering the cooperation cost, it is evident to see that the sales quantity and profit of pharmaceutical supply chain under cooperative decision-making are much higher than those under independent decision-making. This conclusion vividly indicates that winwin cooperation is the future main-strain road of pharmaceutical supply chain. However, as a result of the asymmetry of information and the egoism, the existence of cooperation cost will always be. Exactly on the basis of this fact, this paper sets the unit cooperation cost $C_{0}$, and conforms to the fact that the total income of the supply chain under the cooperative decision is greater than that under the independent decision. So that $C_{0}$ is obtained a value range. Eventually, within this range, consider two conditions. The one is that every supply chain member obtains greater benefits allocated by the pharmaceutical supply chain under cooperative decision than when they make independent decisions. The other is that considering the relative leadership of supply chain members. Then cooperative benefits of pharmaceutical supply chain are reasonably distributed by means of the cubic distribution method based on profit distribution factor.

\section{Conclusion}

By the cubic distribution method based on profit distribution factors, the total cooperative income can be reasonably distributed by pharmaceutical manufacturers, pharmaceutical distributors, third-party logistics service providers and pharmaceutical retailers. The distributed income of each member is higher than that of independent decision, so as to ensure the basis of pharmaceutical supply chain cooperation. According to the calculation results in the paper, the allocation factors $e_{1}, e_{2}$ and $e_{3}$ are in a clear value range. They are in require of further research in order to explore how to gradually narrow this value range until the final result is determined. At the same time, because the cooperation income will 
gradually decrease with the increase of cooperation cost, so we should make full use of information technology to reduce information asymmetry, strengthen the communication, coordination and cooperation mechanism between upstream and downstream of the supply chain, and continuously reduce the cooperation cost, so as to improve the total income of supply chain cooperation. Once the total income of cooperation increases, supply chain members will have more initiative to cooperate.

At last, when dealing with the expense of the third-party logistics service provider in the research process, this paper merely considers the situation borne by the pharmaceutical distributor. If the pharmaceutical manufacturer and the pharmaceutical distributor pay a certain proportion of the logistics expense, how much this proportion will be can motivate the pharmaceutical manufacturer, the pharmaceutical distributor and the third-party logistics service provider to cooperate fully, which will be a topic worthy of research. In modeling, it is assumed that the market demand is the order quantity of retail terminals, but in practice, the demand is actually uncertain. It is a very meaningful research direction to study the cooperative benefit distribution of pharmaceutical supply chain under uncertain demand. In addition, the competitive relationship among pharmaceutical manufacturers, distributors, retailers and third-party logistics service providers has not been considered in the model in this paper. If there are multiple pharmaceutical manufacturers, distributors, retailers or third-party logistics service providers, there is a competitive relationship among them. Then how the supply chain should cooperate and how to distribute the benefits of cooperation need to be further discussed.

\section{Fund}

This research was funded by the enterprise practice training funding project for young teachers of Jiangsu Province (No. 2020QYSJ206).

\section{Conflicts of Interest}

The authors declare no conflicts of interest regarding the publication of this paper.

\section{References}

Chen, X. F., Cai, G. S., \& Song, J. S. (2019). The Cash Flow Advantages of 3PLs as Supply Chain Orchestrators. Manufacturing \& Service Operations Management, 21, 435-451. https://doi.org/10.1287/msom.2017.0667

Chen, X., Zhang, W., \& Gu, W. (2020) Research on the Coordination Model of Pharmaceutical Supply Chain Considering Technology R\&D and Logistics Distribution. Chinese Journal of Management Science, 28, 80-92.

Deng, J., \& Wen, Z. (2018). Blue Book of Pharmaceutical Distribution Industry: Annual Report on China's Pharmaceutical Distribution Industry (2018). Social Sciences Academic Press.

Deng, J., \& Wen, Z. (2019). Blue Book of pharmaceutical Distribution Industry: Annual 
Report on China's Pharmaceutical Distribution Industry (2019). Social Sciences Academic Press.

Di, R., Zhao, Y., \& Yu, H. (2017). Research on Supply Chain Management Optimization of Pharmaceutical Distribution Enterprises Based on Shapley Value. Technical Economics and Management Research, No. 7, 94-96.

Gao, J., Yang, X., \& Liu, D. (2017) Uncertain Shapley Value of Coalitional Game with Application to Supply Chain Alliance. Applied Soft Computing, 56, 551-556. https://doi.org/10.1016/j.asoc.2016.06.018

Giri, B. C., \& Sarker, B. R. (2017). Improving Performance by Coordinating a Supply Chain with Third Party Logistics Outsourcing under Production Disruption. Computers \& Industrial Engineering, 103, 168-177. https://doi.org/10.1016/j.cie.2016.11.022

Gong, Y., Li, B., \& Liu, T. (2008). Pricing and Coordination Strategies for Supply Chain Based on Third-Party Logistics Provider. Journal of Business Research, No. 11, 162-165.

Guan, Y., \& Huang, Z. (2021). Research on Drug Retail Dual-Channel Pricing Strategy Considering Medical Insurance Payment Policy. Journal of China pharmacy, 32, 19261932.

Hafezalkotob, A., Khodabakhsh, M., Saghaei, A., \& Eshghipour, M. (2018). Cooperation of Advertising Companies in Social Networks: A Graph and Game Theory Approaches. Computers \& Industrial Engineering, 125, 212-220. https://doi.org/10.1016/j.cie.2018.08.023

Hou, W., \& Yang, D. (2019). Competition Strategy of Two-Channel Pharmaceutical Supply Chain Based on Medical Insurance Regulation and Consumer Utility. Operations Research and Management Science, 28, 8-15.

Huang, C., Mi, X., \& Kang, B. (2021). Basic Probability Assignment to Probability Distribution funCtion Based on the Shapley Value Approach. International Journal of Intelligent Systems, 36, 4210-4236. https://doi.org/10.1002/int.22456

Lai, X., \& Nie, J. (2021). The Influence of Retailer Service Capability on the Dual-channel Strategy of OTC Drug Supply Chain. Industrial Engineering and Management, 16, 131-139.

Li, S., Dan, B., Zhou, M., \& Wang, D. (2019). Pricing and Coordination Strategies in Pharmaceutical Dual-Channel Supply Chain under the Influence of Price Cap Policy and Public Welfare. Journal of Industrial Engineering/Engineering Management, 33, 196-204.

Liang, P., \& Li, J. (2013). Research on Benefit Distribution Mechanism of Agricultural Supply Chain Alliance Based on Shapley Value Method. Journal of Business Research, No. 8, 191-194+216.

Liu, N., Wu, Q., Lu, Q., \& Pang, H. (2011) Research on Coordination Strategy of TwoLevel Supply Chain with the Logistic Service Provider Involved. Soft Science, 25, 125-129.

Lyu, H., \& Liu, W. (2010) Research on Cooperation Mode and Benefit Distribution Mechanism of Pharmaceutical Supply Chain. Modern Management Science, No. 7, 94-96.

Sha, J., \& Zheng, S. (2021). Revenue Distribution of Hybrid Channel Supply Chain Based on Modified Shapley Value with Cost. Discrete Dyanmics in Nature and Society, 2021, Article ID 440518. https://doi.org/10.1155/2021/4405185

Shi, Y., Zhang, A., Arthanari, T., Liu, Y., \& Cheng, T. C. E. (2016) Third-Party Purchase: An Empirical Study of Third-Party Logistics Providers in China. International Journal of Production Economics, 171, 189-200. https://doi.org/10.1016/j.ijpe.2015.08.028

Volland, J., Fügener, A., Schoenfelder, J., \& Brunner, J. O. (2017) Material Logistics in Hospitals: A Literature Review. Omega, 69, 82-101. 
https://doi.org/10.1016/j.omega.2016.08.004

Xu, M., Yu, G., \& Zhang, H. (2006). Game Analysis in a Supply Chain with Service Provision. Jouranal of Management Sciences in China, 9, 18-28.

Xu, Z., Peng, Z., Yang, L., \& Chen, X. (2018). An Improved Shapley Value Method for a Green Supply Chain Income Distribution Mechanism. International Journal of Environmental Research and Public Health, 15, Article No. 1976. https://doi.org/10.3390/ijerph15091976

Yang, D., \& Hou, W. (2019). Dual Channel Pricing Strategy of Pharmaceutical Supply Chain Considering Channel Competition and Medical Insurance Policy. Chinese Journal of Management, 16, 932-938.

Yang, H. X., Sun, X. L., Liu, W. M., \& Hao, J.-Q. (2017). Optimization of Profit Distribution Method under Multifactor in Construction Supply Chain. Journal of Interdisciplinary Mathematics, 20, 1435-1440. https://doi.org/10.1080/09720502.2017.1382153

Yang, H., Liu, R., \& Li, C. (2017). Benefit Distribution of Agricultural and Supermarket Docking Supply Chain Based on Shapley Value Method and TOPSIS Method. Journal of Jiangsu Agricultural Science, 45, 358-362.

Zheng, H., Song, B., Wu, J., Zhang, N., \& Gao, S. (2018). Investigation and Research on the Influence of "Two-Invoice System" Policy on Pharmaceutical Distribution Enterprises. Journal of China Pharmacy, 29, 2305-2310.

Zheng, Y., Zhang, S., Chen, X., \& Liu, F. (2011). Application of Modified Shapley Value in Gains Allocation of Closed-Loop Supply Chain under Third-Party Reclaim. Energy Procedia, 5, 980-984. https://doi.org/10.1016/j.egypro.2011.03.173

Zhou, L., Cui, H., Huang, C., Kang, B., Zhang, J. (2021). Counter Deception in Belief Functions Using Shapley Value Methodology. International Journal of Fuzzy Systems, 24, 340-354. https://doi.org/10.1007/s40815-021-01139-1

Zhou, Y. (2017) Benefit Distribution Mechanism of Agricultural Supply Chain Based on Improved Shapley Value Model. Statistics and Decision making, 23, 52-54. 